\title{
Mesoscopic g-factor renormalization for electrons in III-V interacting nanolayers
}

\author{
M. A. Toloza Sandoval, J. E. Leon Padilla, and A. Ferreira da Silva \\ Instituto de Física, Universidade Federal da Bahia, 40210-340 Salvador, Bahia, Brazil \\ E. A. de Andrada e Silva \\ Instituto Nacional de Pesquisas Espaciais, 12201-970 São José dos Campos, São Paulo, Brazil \\ G. C. La Rocca \\ Scuola Normale Superiore and CNISM, Piazza dei Cavalieri 7, I-56126 Pisa, Italy
}

(Received 8 June 2018; revised manuscript received 15 July 2018; published 28 August 2018)

\begin{abstract}
The physics of the renormalization of the effective electron $g$ factor by the confining potential in semiconductor nanostructures is theoretically investigated. The effective $g$ factor for electrons in structures with interacting nanolayers, or coupled quantum wells (QWs), is obtained with an analytical and yet accurate multiband envelopefunction solution, based on the linear $8 \times 8 \mathrm{k} \cdot \mathrm{p}$ Kane model for the bulk band structure. Both longitudinal and transverse applied magnetic fields are considered and the g-factor anisotropy (i.e., the difference between the two field configurations) is analyzed over the entire space spanned by the two structure parameters: the thickness of the active layers and the thickness of the tunneling barrier separating them. Two-dimensional anisotropy maps are constructed for symmetric and asymmetric InGaAs coupled QWs, with InP tunneling barriers, that reproduce exactly known single-layer or QW results, in different limits. The effects of the structure inversion asymmetry on the mesoscopic g-factor renormalization are also discussed, in particular the negative anisotropies for thin-layer structures. Such multilayer structures form an excellent testing ground for the theory, and the analytical solution presented, which is perfectly consistent over the whole space of parameters, leads to helpful expressions and can guide further research on the mechanisms of this mesoscopic renormalization.
\end{abstract}

DOI: 10.1103/PhysRevB.98.075312

\section{INTRODUCTION}

Simple GaAs/AlGaAs quantum wells (QWs) represent the ultimate testing system for quantum confinement effects and fundamental methods in condensed-matter physics. For instance, the energy quantization and the validity of the envelope-function approximation have been verified in these nanostructures with great accuracy [1]. Semiconductor QWs support two-dimensional electron gases (2DEGs) and are applied in a large number of electronic devices, in particular lasers and photodetectors, with operation frequencies tuned by the well width $L_{w}$ [2]. More recently, high-quality structures with tunnel-coupled QWs have been fabricated and used in problems and applications in the physics of exciton liquids [3], topological transitions [4], and special field-effect transistors [5], for example. Considering the 1D dynamics along the growth axis, a double quantum well (DQW) behaves with respect to the constituent QWs similarly to a biatomic molecule with respect to the atoms. These DQW structures support interacting 2DEGs and allow for tunneling effects used, for instance, to control the charge transfer between the active layers. With respect to single QWs, DQWs form more general quantum structures with at least two independent parameters: $L_{w}$ and $L_{b}$, the active layers' width and that of the barrier in between, which is the inverse of the tunnel-coupling parameter. The single QW limit of the DQW structures, both when $L_{b}=0$ and when it is sufficiently large (so that the interwell coupling is negligible), is a condition of particular interest in the modeling of fine quantum confinement effects, as the g-factor renormalization and the corresponding Zeeman splitting in such nanostructures.

Due to the increasing interest in spintronics [6] and in new schemes for quantum computation, including the detection of Majorana fermions [7], much attention has been given lately to the renormalization of the electron $\mathrm{g}$ factor in semiconductor nanostructures [8-18]. The mesoscopic confining potential in semiconductor nanostructures further renormalizes the bulk effective $\mathrm{g}$ factor (already renormalized from the bare value 2) and introduces extra anisotropies, transforming scalar g factors into tensors. The g-factor engineering starts to be a fundamental part of semiconductor physics [19]; however, it is still largely based only on the Roth formula for the bulk [20] and, before achieving the desired control in nanostructures (quantum wells, wires, etc.), the problems found when modeling and measuring this mesoscopic renormalization in simple III-V QWs need to be solved.

After the work of many groups [21-40], it is by now well established that the ground-state effective $\mathrm{g}$ factor for electrons confined in regular GaAs-like $\mathrm{QWs}\left(g^{\mathrm{QW}}\right)$ varies with $L_{w}$ interpolating from the bulk barrier (AlGaAs) to the bulk well (GaAs) g factors, when $L_{w}$ goes from zero to a sufficiently large value; and that between these two bulk limits, $g^{\mathrm{QW}}\left(L_{w}\right)$, depends on the magnetic-field orientation. The difference in the QW $\mathrm{g}$ factor between the magnetic-field orientations perpendicular and parallel to the interfaces gives the g-factor main anisotropy $\Delta g^{\mathrm{QW}}\left(=g_{\|}-g_{\perp}\right)$ which is the most direct 
and critical signature of the quantum confinement, and has been much investigated both theoretically $[13,14,17,22,41]$ and experimentally $[8,24,26,32,39]$. QW structures made out of different compounds were investigated and it is known, for example, that except for very thin asymmetric wells [13], electrons in GaAs-like QWs have a positive anisotropy, i.e., $g_{\|}>g_{\perp}$, where $g_{\|}$refers to an in-plane magnetic field configuration, while $g_{\perp}$ to a magnetic field along the growth direction (note, however, that the opposite definition of anisotropy is also used in the literature). The $L_{w}$ dependence of the anisotropy is more interesting. In symmetric QWs, the anisotropy must satisfy strict bulk limits that impose $\Delta g^{\mathrm{QW}}\left(L_{w}=0\right)$ and $\Delta g^{\mathrm{QW}}\left(L_{w} \rightarrow \infty\right)$ both exactly zero, and present a critical $L_{w}$ with maximum (or minimum) $\Delta g^{\mathrm{QW}}$. However it has not been easy to observe and verify these properties. Experimentally, special conditions for the electron spin resonance [24] or sophisticated time-resolved techniques $[26,32]$ have been necessary and, nevertheless, $\Delta g^{\mathrm{QW}}$ has been measured only with large uncertainties and a small number of samples.

From the theoretical point of view, despite the need of corrections due to the remote bands, the envelope-function approximation based on the eight-band Kane model remains the most promising approach, in particular to obtain general results and expressions, with simple physical interpretations. However, with this same approach, there are different approximate solutions for the effective $\mathrm{g}$ factor [22,24,31,41]. The simple analytical solution proposed in Ref. [41] (see also Ref. [13] for details) has the advantages of satisfying exactly the above-mentioned bulk conditions in $\Delta g^{\mathrm{QW}}$ and of leading to useful expressions, but has been applied only to single layers or QWs. The extension and application of such solution to more complex and general structures, including, for example, competing structure inversion asymmetry (SIA) and tunneling effects, is a critical test of the theory and a clear step forward in our understanding of the mesoscopic g-factor renormalization.

This is what we do here: a detailed and complete solution for the electron effective $\mathrm{g}$ factor in symmetric and asymmetric III-V interacting layers or DQW structures. The obtained results are perfectly coherent over the entire space of the structure parameters, exactly reproduce known single QW results in the two corresponding limits, and include helpful expressions and recipes for the estimation/calculation of the effective $g$ factor in general III-V nanostructures. The interacting features observed between the noninteracting (single QW) limits, as well as the effects of SIA, are simply explained in terms of the Rashba spin-orbit coupling and the electron wave function in these structures. As a specific example, 2D anisotropy maps $\Delta g^{\mathrm{DQW}}\left(L_{w}, L_{b}\right)$ are constructed for symmetric $\mathrm{InP} / \mathrm{InGaAs} / \mathrm{InP} / \mathrm{InGaAs} / \mathrm{InP}$ and asymmetric insulator/InGaAs/InP/InGaAs/InP multilayer structures, and discussed in detail. Next we present and discuss the model calculation and then the results, before giving a summary of the main conclusions.

\section{THEORETICAL MODEL}

A general anisotropic Zeeman response to an applied magnetic field $\vec{B}$ is described by an effective g-factor tensor $g_{i j}^{*}$ defined by the following Zeeman term in the electron effective
Hamiltonian:

$$
\mathcal{H}_{Z}^{*}=\frac{\mu_{0}}{2} \sigma_{i} g_{i j}^{*} B_{j},
$$

with $i, j=x, y, z, \vec{\sigma}=\left(\sigma_{x}, \sigma_{y}, \sigma_{z}\right)$ being the Pauli matrices spin vector, and $\mu_{0}=e \hbar / 2 m_{0}$ the Bohr magneton, with $e$ and $m_{0}$ being the free-electron charge and mass. In 2D structures (QWs, DQWs, etc.) of the nine independent elements of $g^{*}$, only two are nonzero in the first order: $g_{\|}$and $g_{\perp}$, diagonal elements corresponding to magnetic fields parallel and perpendicular, respectively, to the interfaces. In this approximation, we neglect the much smaller off-diagonal elements, which include the in-plane anisotropy. We also neglect the bulk k-cubic Dresselhaus spin-orbit coupling contribution, which in these InGaAs QWs is much smaller than the Rashba one $[8,42,43]$.

Starting with the applied magnetic field $\vec{B}$ parallel to the interfaces (say in the $y$ direction, with the growth direction along $z$ ), the following effective Hamiltonian for electrons in 2D structures can be easily derived from the linear $8 \times 8$ Kane model [41]:

$$
\begin{aligned}
\mathcal{H}(z, \varepsilon)= & -\frac{\hbar^{2}}{2} \frac{d}{d z} \frac{1}{m(z, \varepsilon)} \frac{d}{d z}+\frac{1}{2} \frac{m_{0}^{2}}{m(z, \varepsilon)} \omega_{c}^{2}\left(z-z_{c}\right)^{2} \\
& +E_{c}(z)+\frac{\mu_{0}}{2} \sigma_{y}\left\{g_{0}-\frac{4 m_{0}}{\hbar^{2}}[\beta(z, \varepsilon)\right. \\
& \left.\left.+\left(z-z_{c}\right) \frac{d}{d z} \beta(z, \varepsilon)\right]+\delta g_{\text {rem }}(z)\right\} B
\end{aligned}
$$

All different terms above are easily recognized, the last one being the effective Zeeman interaction; $g_{0}(=2)$ is the bare electron $\mathrm{g}$ factor, $\omega_{c}=e B / m_{0}$ is the cyclotron frequency, $\beta(z, \varepsilon)$ is the spin-orbit coefficient, $\frac{d}{d z} \beta=\alpha_{R}$ is the so-called Rashba coupling parameter [44-46], $\delta g_{\text {rem }}(z)$ is the remote band's correction [47], and $z_{c}=-\ell^{2} k_{x}$ is the center of the cyclotron orbit with magnetic length $\ell=\sqrt{\hbar / e B}$ (note that the Hamiltonian does not depend on $x$, so that a plane wave was chosen for the $x$-dependent part of the wave function). In addition, $E_{c}(z)$ describes the conduction-band-edge profile and, for completeness, we recall that the energy-dependent effective mass and spin-orbit coefficient are given by

$$
\frac{1}{m(z, \varepsilon)}=\frac{P^{2}}{\hbar^{2}}\left[\frac{2}{\varepsilon-E_{v}(z)}+\frac{1}{\varepsilon-E_{v}(z)+\Delta(z)}\right]
$$

and

$$
\beta(z, \varepsilon)=\frac{P^{2}}{2}\left[\frac{1}{\varepsilon-E_{v}(z)}-\frac{1}{\varepsilon-E_{v}(z)+\Delta(z)}\right],
$$

where $P$ is the Kane matrix element, $E_{v}(z)\left[=E_{c}(z)-E_{g}(z)\right]$ stands for the valence-band edge profile, and $\Delta$ is the valenceband spin-orbit energy splitting. Note that in the flat-band case considered here, $\beta$ is a piecewise constant function of $z$, changing at each heterostructure interface where $\alpha_{R}$ behaves as a $\delta$ function.

It is easy to check that the above effective Hamiltonian (2) exactly satisfies three well-known and fundamental limits: bulk (no $z$ dependence), no spin orbit $(\beta=0)$, and zero field $(B=$ 0 ). More important here though is that in the limit of $B \rightarrow 0, g_{\|}$ can be obtained in first-order perturbation theory. One simply computes the expectation value $\left\langle\mathcal{H}_{Z}^{*}\right\rangle_{\psi_{0}}$ with the unperturbed 

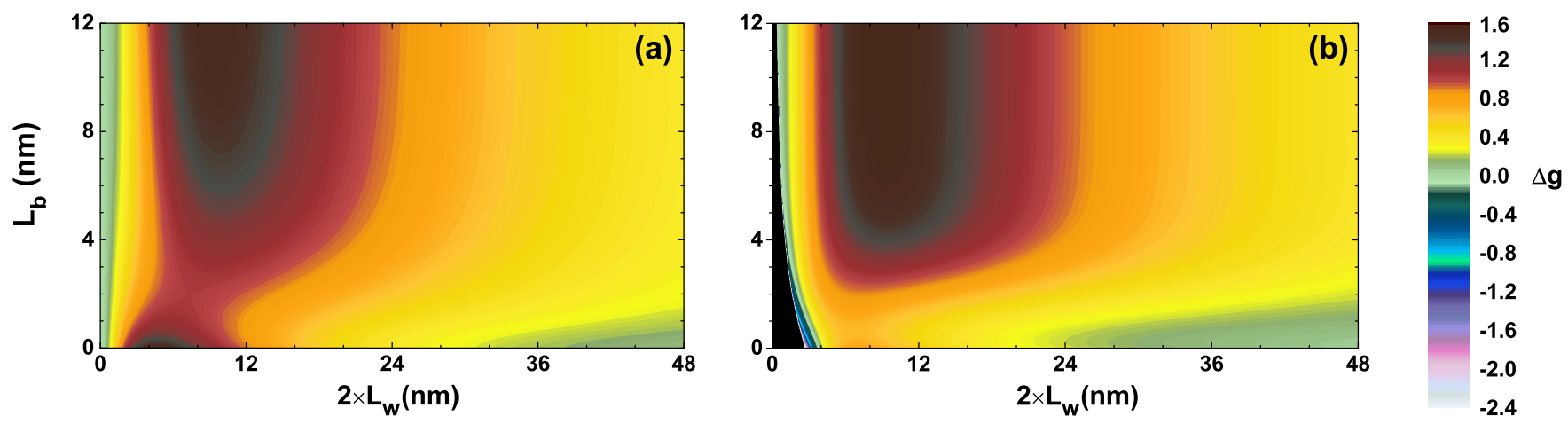

FIG. 1. 2D maps of the g-factor anisotropy for electrons in (a) symmetric and (b) asymmetric InGaAs/InP DQW structures. The anisotropy is given by the color code on the right. Among other features discussed in the text, note the large negative values of the anisotropy in asymmetric structures with very thin layers, in the lower left angle of the map, right next to the black area on the left, which is the region with no bound states allowed. For the band profile and parameters, please see Figs. 2 and 3.

eigenstate $\psi_{0}$ (with energy $\varepsilon_{0}$ ). The unperturbed problem is that for $B=0$, i.e., $\mathcal{H}^{*}(B=0)\left|\psi_{0}\right\rangle=\varepsilon_{0}\left|\psi_{0}\right\rangle$. By recalling that in an undoped (flat-band) structure $\beta(z)$ is given by a step function, with discontinuous jumps at each interface (where it changes from one bulk value to another), one clearly sees that $g_{\|}=\left\langle g_{\text {bulk }}\right\rangle_{\psi_{0}}+\Delta g^{2 D}$, i.e., the sum of the bulk average plus an interface contribution [41],

$$
\Delta g^{2 D}=\left(4 m_{0} / \hbar^{2}\right)\left\langle\alpha_{R}\left(z, \varepsilon_{0}\right)\left(z_{0}-z\right)\right\rangle_{\psi_{0}},
$$

where $z_{0}=\langle z\rangle_{\psi_{0}}$. Even for 2D structures with band profiles far from flat, there is always the $\alpha_{R} \neq 0$ contribution at the interfaces, which is proportional to $\delta \beta=\beta_{w}-\beta_{b}$, the only one for flat bands and often the dominant one. Since the bulk average is independent of the field and/or growth orientation, $\Delta g^{2 D}$ is identified as the g-factor anisotropy because, in the same order of approximation, one finds $g_{\perp}=\left\langle g_{\text {bulk }}\right\rangle_{\psi_{0}}$. Despite the larger symmetry, the theory for the perpendicular configuration is less straightforward [13,31]; nevertheless, the result could not be simpler, more reasonable, and in better agreement with the experiments $[23,25,26,32,36]$. In a more usual form, the averaged $g_{\text {bulk }}$ can be written in terms of the main parameters as

$$
g_{\perp}=g_{0}-\sum_{i=b, w}\left[\frac{m_{0}}{m_{i}\left(\varepsilon_{0}\right)} \frac{2 \Delta^{(i)}}{3 \varepsilon_{g}^{(i)}\left(\varepsilon_{0}\right)+2 \Delta^{(i)}}-\delta g_{r}^{(i)}\right] P_{i},
$$

where $P_{i}$ is the probability to find the electron in the layers $i$ (well or barrier). It is an expectation value of the bulk $\mathrm{g}$ factor calculated, however, with an (energy-dependent) effective gap $\varepsilon_{g}^{(i)}\left(\varepsilon_{0}\right)\left(=\varepsilon_{0}-E_{c}^{(i)}+E_{g}^{(i)}\right)$; this formula generalizes that of Roth et al. for the bulk [20] and is part of our analytical solution for the g-factor renormalization in 2D structures. It is particularly useful when the electron density of probability is concentrated in the active layers only, so that $g_{\perp}$ is determined by the confinement energy shift only, as already observed experimentally [36]. The known bulk formula is recovered in limits of $L_{w} \rightarrow 0$ and of sufficiently large $L_{w}$, when $\varepsilon_{g}^{b}\left(\varepsilon_{0}\right) \rightarrow$ $E_{g}^{b}$ (with $P_{w}=0$ and $P_{b}=1$ ) and $\varepsilon_{g}^{w}\left(\varepsilon_{0}\right) \rightarrow E_{g}^{w}$ (with $P_{w}=1$ and $P_{b}=0$ ), respectively [note that the energy-dependent effective mass also goes to the bulk band-edge effective mass, i.e., in these bulk limits, one has, respectively, $m_{b}\left(\varepsilon_{0}\right)=m_{b}^{*}$ and $m_{w}\left(\varepsilon_{0}\right)=m_{w}^{*}$.

\section{RESULTS AND DISCUSSION}

As a practical example, let us consider InP/InGaAs/InP/ InGaAs/InP symmetric DQWs and insulator/InGaAs/InP/ InGaAs/InP asymmetric DQWs structures, which depend on the same set of parameters, given by the width of the InGaAs and (middle) InP nanolayers, $L_{w}$ and $L_{b}$, plus their bulk band parameters and band offset, and can then be better compared in order to assess the effects of SIA on this mesoscopic renormalization.

The recipe is then as follows: for each point in the space spanned by the two varying parameters, $L_{w}$ and $L_{b}$, we (1) solve the unperturbed problem to obtain $\psi_{0}$ and $\varepsilon_{0}$, the wave function and energy of the ground state of the Kane's DQW problem; then, (2) calculate $z_{0}=\langle z\rangle_{\psi_{0}}$ and the probabilities $P_{i}=\int_{i}\left|\psi_{0}\right|^{2} d z$; and, finally, (3) substitute them into the equations above and get $\Delta g^{\mathrm{DQW}}, g_{\perp}^{\mathrm{DQW}}$, and $g_{\|}^{\mathrm{DQW}}$.

With a color code, Fig. 1 shows the obtained anisotropy maps for symmetric [Fig. 1(a)] and asymmetric [Fig. 1(b)] DQW structures, showing rich landscapes with qualitative differences, particularly in the strong-coupling regime at the small- $L_{b}$ region. Note first that for the symmetric structures (SDQWs), the $L_{w}$ dependence of the anisotropy for different values of $L_{b}$ always satisfies the bulk conditions; i.e., independent of $L_{b}$, the g-factor anisotropy goes indeed to zero as $L_{w} \rightarrow 0$ or $\infty$, and that $\Delta g$ presents always a single maximum, limited though by the maximum $\Delta g^{\mathrm{QW}}$. By increasing the width of the InP barrier between the InGaAs nanolayers, a continuous crossover from one noninteracting single QW limit to another is obtained (i.e., from a $2 L_{w}$ wide $\mathrm{QW}$ to an $L_{w}$ one); one exactly obtains $\Delta g^{\mathrm{SDQW}}\left(L_{w}, L_{b}=0\right)=$ $\Delta g^{\mathrm{QW}}\left(2 L_{w}\right)$ and $\Delta g^{\mathrm{SDQW}}\left(L_{w}, L_{b} \rightarrow \infty\right)=\Delta g^{\mathrm{QW}}\left(L_{w}\right)$.

Probably the most interesting difference seen in asymmetric DQWs is the large negative anisotropies (down to $\Delta g=-2.4$ ) seen in the small area at the lower-left angle of the map, corresponding to thin-layer structures, just on the right of the black area, which is the region of the asymmetric structures with no allowed bound states. The differences in $\mathrm{g}$-factor anisotropy 


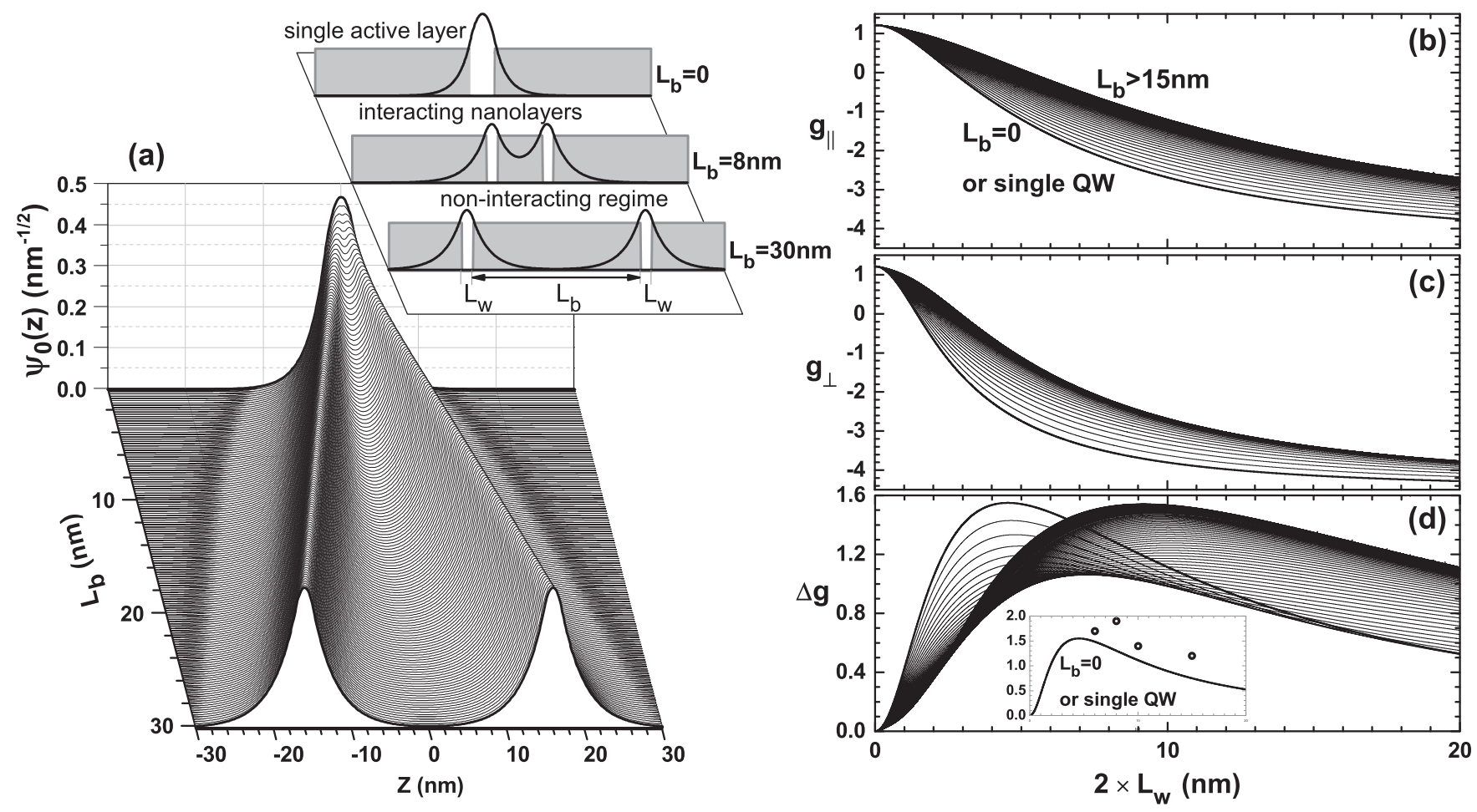

FIG. 2. Detailed results for the effective $\mathrm{g}$ factor of electrons in InGaAs/InP symmetric DQW structures, illustrated in the inset, with examples of the three different interacting regimes, corresponding to interwell barrier width $L_{b}$ zero, intermediate, and sufficiently large; $L_{w}=2 \mathrm{~nm}$. Layers of InP and $\operatorname{In}_{0.53} \mathrm{Ga}_{0.47}$ As are considered with the following parameters: $E_{g}^{\operatorname{In} P}=1.424 \mathrm{eV}, \Delta_{s o}^{\operatorname{In} P}=0.108 \mathrm{eV}, g_{\text {In } P}^{*}=1.2$; and $E_{g}^{\mathrm{InGaAs}}=0.813 \mathrm{eV}, \Delta_{s o}^{\mathrm{InGaAs}}=0.326 \mathrm{eV}, g_{\mathrm{InGaAs}}^{*}=-4.5$, and $m_{\mathrm{InGaAs}}^{*}=0.041 m_{e}$; [48] with a conduction-band offset of $0.25 \mathrm{eV}$ [49]. It is plotted in (a) $\psi_{0}(z)$, and, as a function of $L_{w}, g_{\perp}, g_{\|}$, and $\Delta g$, in (b)-(d), respectively. In (a), $L_{b}$ varies from zero (on top) up to $30 \mathrm{~nm}$ (bottom) in units of $0.2 \mathrm{~nm}$, used also in the other plots. In (d), one sees the anisotropy $\Delta g^{\mathrm{SDQW}}$ interpolating from one single QW limit to another, as $L_{b}$ goes from zero up to a sufficiently large value, as discussed in the text, and the inset shows the comparison with available experimental data [24].

between SDQWs and ADQWs are better understood with their corresponding explicit expressions. In flat-band structures, the expectation value in Eq. (5) is easily computed and $\Delta g^{2 D}$ is seen to be given in terms of the density of probability $\mathcal{P}$ $\left(=\left|\psi_{0}\right|^{2}\right)$ at the different interfaces. In SDQWs, there are only two nonequivalent interfaces, called 1 and 2 , and one gets

$$
\Delta g^{\mathrm{SDQW}}=\left[\left(\mathcal{P}_{2}-\mathcal{P}_{1}\right) L_{b}+2 \mathcal{P}_{2} L_{w}\right] \frac{4 m_{0} \delta \beta}{\hbar^{2}},
$$

with the $z$ origin taken at the middle of the structure (see Fig. 2); thus, $z_{0}=0, \mathcal{P}_{1}=\left|\psi_{0}\left(L_{b} / 2\right)\right|^{2}$, and $\mathcal{P}_{2}=\left|\psi_{0}\left(L_{w}+L_{b} / 2\right)\right|^{2}$. In the ADQWs considered here, instead, with the $z$ origin taken at the leftmost infinite barrier with $\left|\psi_{0}(z=0)\right|^{2}=0$ (see Fig. 3), three nonequivalent interfaces contribute (at $z=$ $L_{w}, L_{w}+L_{b}, 2 L_{w}+L_{b}$ ), and numbering them from the left, one gets

$$
\begin{aligned}
\Delta g^{\mathrm{ADQW}}= & {\left[\left(\mathcal{P}_{3}-\mathcal{P}_{2}+\mathcal{P}_{1}\right)\left(L_{w}-z_{0}\right)\right.} \\
& \left.+\left(\mathcal{P}_{3}-\mathcal{P}_{2}\right) L_{b}+\mathcal{P}_{3} L_{w}\right] \frac{4 m_{0} \delta \beta}{\hbar^{2}} .
\end{aligned}
$$

These expressions complete the analytical solution and make clear each feature of the maps in Fig. 1, with a look at the corresponding $\psi_{0}$, as well as at the individual elements $g_{\perp}$ and $g_{\|}$. See, for instance, the results in Figs. 2 and 3 for $L_{w}=2 \mathrm{~nm}$ SDQWs and ADQWs, respectively, with varying
$L_{b}$ in a fine grid $(0.2 \mathrm{~nm})$, where (a) shows $\psi_{0}(z)$, (b) $g_{\perp}$, (c) $g_{\|}$, and (d) $\Delta g$, and the insets give examples of the three different interacting regimes, i.e., the noninteracting single $\mathrm{QW}$, in the limits of $L_{b}=0$ and $L_{b}>>L_{w}$, and the coupled regime in between.

The SDQW and ADQW $\psi_{0}$ are quite different; in ADQWs, $\left|\psi_{0}\right|^{2}$ is always concentrated in only one layer (that on the right, far from the large barrier on the left), while in SDQWs, it splits equally between the two. To this corresponds an important qualitative difference also in $z_{0}$ as a function of $L_{w}$ and $L_{b}$; while it is always zero in SDQWs, in ADQWs instead it does vary significantly and is responsible for the negative anisotropies in very thin-layer structures. In such thin-layer limit, $\psi_{0}$ describes states with energy close to the top of the InP barrier, near the region with no allowed bound states, and strongly feels the asymmetry of the confining potential. For this reason, the SDQW and $\mathrm{ADQW}$ results in this regime present larger contrast, and the ADQW $z_{0}$ is pushed away from the larger barrier, leading to the negative anisotropies.

Experimentally, the g-factor renormalization in InGaAs nanolayers has been investigated by Kowalski et al. [24] and, more recently, also by Herzog et al. [8]. The large confinementinduced anisotropy of the $\mathrm{g}$ factor in InGaAs QWs was confirmed in both studies. Modulation doping structures were used and a direct comparison with theory would require precise knowledge of the structure parameters and its self-consistent 

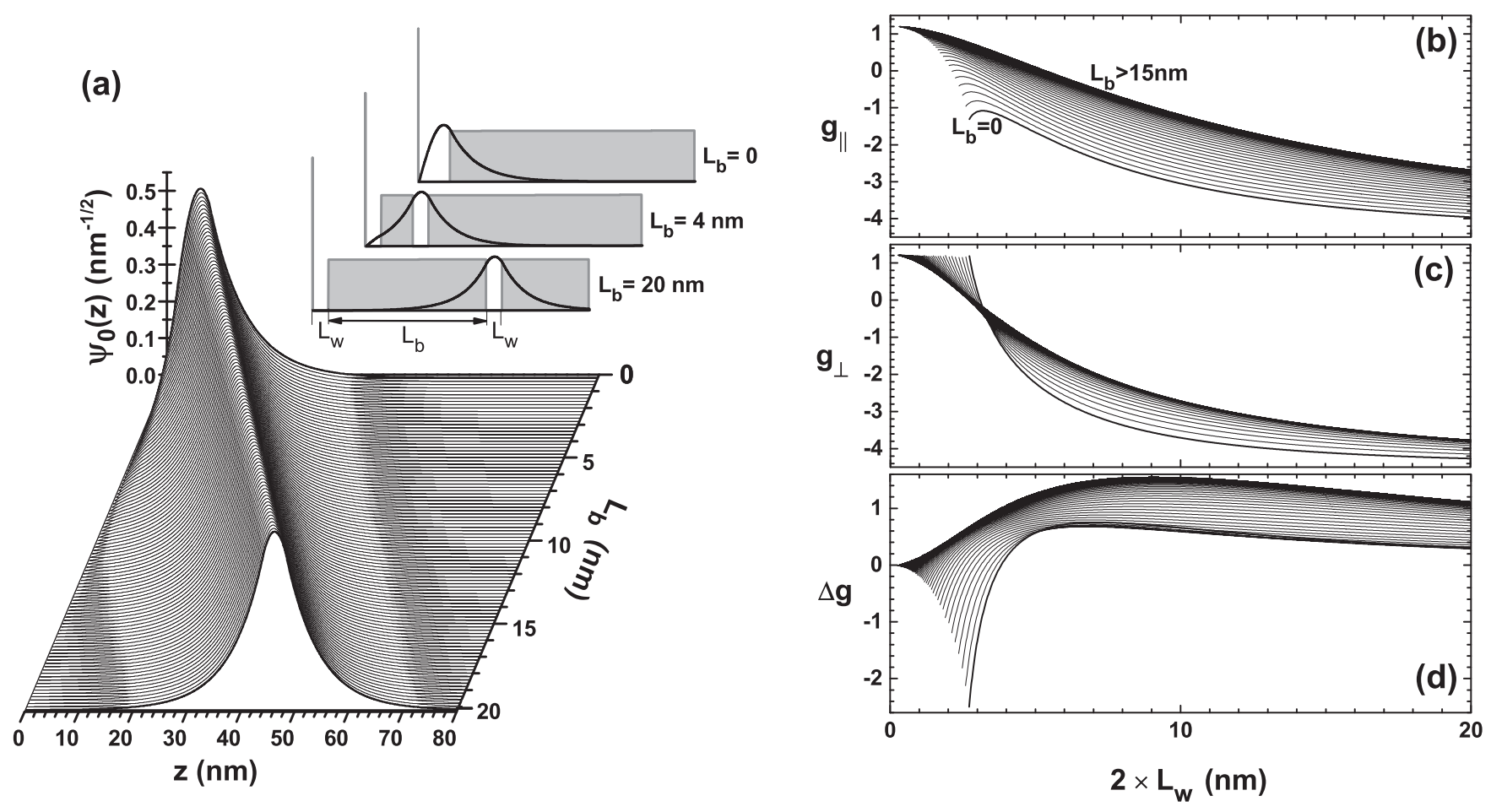

FIG. 3. Unperturbed wave function and renormalized effective $\mathrm{g}$ factors for asymmetric DQW structures of the type insulator/InGaAs/InP/InGaAs/InP, illustrated in the inset. $L_{w}$ and $L_{b}$ give the width of the InGaAs active layers and that of the tunneling InP barrier. The parameters used were as in Fig. 2. The differences seen with respect to the SDQW results derive from the SIA and are explained with the Rashba spin-orbit coupling in these structures (see the discussion in the text).

band profile. Nevertheless, in the inset of Fig. 2(d), we compare our flat-band results with the plain data of Ref. [24] and obtain a reasonably good agreement, as the one already shown for $\mathrm{GaAs}$ systems [41]. Note that the curve in the inset is for symmetric QWs, which, for this experimental $L_{w}$ range, coincide with the results for the asymmetric QWs considered here. However, studies of samples with symmetric and asymmetric InGaAs DQW structures are still necessary in order to fully verify the above predictions for the large g-factor renormalization and anisotropy in these structures with interacting nanolayers.

\section{CONCLUSIONS}

Summarizing, we have solved the g-factor renormalization in III-V semiconductor DQW structures, within the multiband envelope function and perturbation approximations using the $8 \times 8$ Kane model for the bulk. Symmetric and asymmetric structures have been considered. Useful expressions are derived which explain well the available experimental data, and are applied to calculate the main effective g-factor components for electrons in $\mathrm{InP} / \mathrm{InGaAs} / \mathrm{InP} / \mathrm{InGaAs} / \mathrm{InP} \mathrm{SDQWs}$ and
insulator/InGaAs/InP/InGaAs/InP ADQWs. With the resulting g-factor anisotropy as a function of the InGaAs-layer width and the middle InP-layer width, 2D maps were then constructed and compared to assess the effects of SIA on the g-factor mesoscopic renormalization. The qualitative differences are simply explained with the structure's unperturbed wave function $\psi_{0}$. Besides the specific numerical predictions for the InGaAs DQW structures, the overall consistency of the results, shown over the whole space of the structure's parameters, and the general expressions derived give enough ground to believe that the present simple and transparent calculation can guide/help further research to fully understand the mesoscopic renormalization of the electron $\mathrm{g}$ factor in nanostructures.

\section{ACKNOWLEDGMENTS}

E.A.A.S. is thankful to the Scuola Normale Superiore di Pisa for the kind hospitality. Support from the Brazilian agencies FAPESB, FAPESP (Grant No. 2016/03854-9), CAPES, and $\mathrm{CNPq}$ is also acknowledged.
[1] G. Bastard, J. Brum, and R. Ferreira, in Semiconductor Heterostructures and Nanostructures, Solid State Physics, Vol. 44, edited by H. Ehrenreich and D. Turnbull (Academic, New York, 1991), pp. 229-415.
[2] H. Kroemer, Rev. Mod. Phys. 73, 783 (2001).

[3] M. Stern, V. Umansky, and I. Bar-Joseph, Science 343, 55 (2014).

[4] S. S. Krishtopenko, W. Knap, and F. Teppe, Sci. Rep. 6, 30755 (2016). 
[5] X. G. Peralta, S. J. Allen, M. C. Wanke, N. E. Harff, J. A. Simmons, M. P. Lilly, J. L. Reno, P. J. Burke, and J. P. Eisenstein, Appl. Phys. Lett. 81, 1627 (2002).

[6] I. Žutić, J. Fabian, and S. Das Sarma, Rev. Mod. Phys. 76, 323 (2004).

[7] V. Mourik, K. Zuo, S. M. Frolov, S. R. Plissard, E. P. A. M. Bakkers, and L. P. Kouwenhoven, Science 336, 1003 (2012).

[8] F. Herzog, H. Hardtdegen, T. Schäpers, D. Grundler, and M. A. Wilde, New J. Phys. 19, 103012 (2017).

[9] A. Tadjine, Y.-M. Niquet, and C. Delerue, Phys. Rev. B 95, 235437 (2017).

[10] F. Qu, J. van Veen, F. K. de Vries, A. J. A. Beukman, M. Wimmer, W. Yi, A. A. Kiselev, B.-M. Nguyen, M. Sokolich, M. J. Manfra, F. Nichele, C. M. Marcus, and L. P. Kouwenhoven, Nano Lett. 16, 7509 (2016).

[11] X. Mu, G. Sullivan, and R.-R. Du, Appl. Phys. Lett. 108, 012101 (2016).

[12] V. V. Belykh, D. R. Yakovlev, J. J. Schindler, E. A. Zhukov, M. A. Semina, M. Yacob, J. P. Reithmaier, M. Benyoucef, and M. Bayer, Phys. Rev. B 93, 125302 (2016).

[13] M. A. Toloza Sandoval, E. A. de Andrada e Silva, A. Ferreira da Silva, and G. C. La Rocca, Semicond. Sci. Technol. 31, 115008 (2016).

[14] M. Li, Z.-B. Feng, L. Fan, Y. Zhao, H. Han, and T. Feng, J. Magn. Magn. Mater. 403, 81 (2016).

[15] M. Pakmehr, A. Khaetskii, B. D. McCombe, N. Bhandari, M. Cahay, O. Chiatti, S. F. Fischer, C. Heyn, and W. Hansen, Appl. Phys. Lett. 107, 082107 (2015).

[16] L.-W. Yang, Y.-C. Tsai, Y. Li, A. Higo, A. Murayama, S. Samukawa, and O. Voskoboynikov, Phys. Rev. B 92, 245423 (2015).

[17] E. Ridolfi, E. A. de Andrada e Silva, and G. C. La Rocca, Phys. Rev. B 91, 085313 (2015).

[18] A. V. Shchepetilnikov, Y. A. Nefyodov, I. V. Kukushkin, L. Tiemann, C. Reichl, W. Dietsche, and W. Wegscheider, Phys. Rev. B 92, 161301 (2015).

[19] H. Kosaka, A. Kiselev, F. Baron, K. Wook Kim, and E. Yablonovitch, Electron. Lett. 37, 464 (2001).

[20] L. M. Roth, B. Lax, and S. Zwerdling, Phys. Rev. 114, 90 (1959).

[21] T. P. Smith III and F. F. Fang, Phys. Rev. B 35, 7729 (1987).

[22] E. L. Ivchenko and A. A. Kiselev, Sov. Phys. Semicond. 26, 827 (1992).

[23] M. J. Snelling, G. P. Flinn, A. S. Plaut, R. T. Harley, A. C. Tropper, R. Eccleston, and C. C. Phillips, Phys. Rev. B 44, 11345 (1991).

[24] B. Kowalski, P. Omling, B. K. Meyer, D. M. Hofmann, C. Wetzel, V. Härle, F. Scholz, and P. Sobkowicz, Phys. Rev. B 49, 14786 (1994).

[25] R. Hannak, M. Oestreich, A. Heberle, W. Rühle, and K. Köhler, Solid State Commun. 93, 313 (1995).
[26] P. Le Jeune, D. Robart, X. Marie, T. Amand, M. Brosseau, J. Barrau, and V. Kalevcih, Semicond. Sci. Technol. 12, 380 (1997).

[27] E. Ivchenko, A. Kiselev, and M. Willander, Solid State Commun. 102, 375 (1997).

[28] Q. X. Zhao, M. Oestreich, and N. Magnea, Appl. Phys. Lett. 69, 3704 (1996).

[29] A. A. Sirenko, T. Ruf, M. Cardona, D. R. Yakovlev, W. Ossau, A. Waag, and G. Landwehr, Phys. Rev. B 56, 2114 (1997).

[30] A. A. Kiselev, E. L. Ivchenko, and U. Rössler, Phys. Rev. B 58, 16353 (1998).

[31] A. A. Kiselev, K. W. Kim, and E. L. Ivchenko, Phys. Status Solidi (b) 215, 235 (1999).

[32] A. Malinowski and R. T. Harley, Phys. Rev. B 62, 2051 (2000).

[33] R. Kotlyar, T. L. Reinecke, M. Bayer, and A. Forchel, Phys. Rev. B 63, 085310 (2001).

[34] X. C. Zhang, K. Ortner, A. Pfeuffer-Jeschke, C. R. Becker, and G. Landwehr, Phys. Rev. B 69, 115340 (2004).

[35] M. de Dios-Leyva, E. Reyes-Gómez, C. A. Perdomo-Leiva, and L. E. Oliveira, Phys. Rev. B 73, 085316 (2006).

[36] I. A. Yugova, A. Greilich, D. R. Yakovlev, A. A. Kiselev, M. Bayer, V. V. Petrov, Y. K. Dolgikh, D. Reuter, and A. D. Wieck, Phys. Rev. B 75, 245302 (2007).

[37] P. Pfeffer and W. Zawadzki, Phys. Rev. B 74, 115309 (2006).

[38] P. Pfeffer and W. Zawadzki, Phys. Rev. B 74, 233303 (2006).

[39] S. Tomimoto, S. Nozawa, Y. Terai, S. Kuroda, K. Takita, and Y. Masumoto, Phys. Rev. B 81, 125313 (2010).

[40] A. Bruno-Alfonso, F. E. López, N. Raigoza, and E. ReyesGómez, Eur. Phys. J. B 74, 319 (2010).

[41] M. A. Toloza Sandoval, A. Ferreira da Silva, E. A. de Andrada e Silva, and G. C. La Rocca, Phys. Rev. B 86, 195302 (2012).

[42] E. A. de Andrada e Silva, Phys. Rev. B 46, 1921 (1992).

[43] P. S. Eldridge, J. Hübner, S. Oertel, R. T. Harley, M. Henini, and M. Oestreich, Phys. Rev. B 83, 041301 (2011).

[44] L. G. Gerchikov and A. V. Subashiev, Sov. Phys. Semicond. 26, 73 (1992).

[45] E. A. de Andrada e Silva, G. C. La Rocca, and F. Bassani, Phys. Rev. B 50, 8523 (1994).

[46] E. de Andrada e Silva, G. La Rocca, and F. Bassani, Phys. Rev. B 55, 16293 (1997).

[47] The remote bands correction is the difference between the known bulk $\mathrm{g}$ factor and that given by the eight-band Kane model, which is added to the effective Hamiltonian so that it will give the correct $\mathrm{g}$ factor in the two bulk limits of the structure. In InGaAs, it is of $30 \%$ (i.e., 1.36/4.5) and in InP of 20\% (0.24/1.2).

[48] O. Madelung, W. von der Osten, and U. Rössler, in LandoltBornstein New Series, edited by O. Madelung, Vol. 3/22a (Springer, Berlin, 1987).

[49] J. Davies, Physics of Low-dimensional Semiconductors: An Introduction (Cambridge University Press, Cambridge, 1998). 